\title{
Análise do óleo essencial de folhas de três quimiotipos de Lippia alba (Mill.) N. E. Br. (Verbenaceae) cultivados em condições semelhantes
}

\author{
E.S.Tavares ${ }^{1 *}$, L.S. Julião ${ }^{1}$, D. Lopes ${ }^{2}$, H.R. Bizzo ${ }^{2}$, C.L.S. Lage L S.G. Leitão $^{4}$ \\ ${ }^{I}$ Departamento de Botânica, Instituto de Biologia, Universidade Federal do Rio de Janeiro, Rua Brigadeiro \\ Trompovsky s/n, Ilha do Fundão, 21951-590, Rio de Janeiro, RJ, Brasil, \\ ${ }^{2}$ Centro Nacional de Pesquisa de Tecnologia Agroindustrial de Alimentos, EMBRAPA, Av. das Américas, 29501, \\ 23020-470, Rio de Janeiro, RJ, Brasil, \\ ${ }^{3}$ Laboratório de Fisiologia Vegetal, Instituto de Biofisica,Universidade Federal do Rio de Janeiro, Ilha do Fundão, \\ 21941-590, Rio de Janeiro, RJ, Brasil, \\ ${ }^{4}$ Departamento de Produtos Naturais e Alimentos, Faculdade de Farmácia, Universidade Federal do Rio de \\ Janeiro, Ilha do Fundão, 21941-590, Rio de Janeiro, RJ, Brasil
}

\begin{abstract}
RESUMO: A composição do óleo essencial de Lippia alba apresenta variação quantitativa e qualitativa, levando à separação em quimiotipos. O trabalho tem como objetivo analisar o óleo essencial de folhas de três quimiotipos de Lippia alba, provenientes de diferentes regiões do Brasil, cultivados em condições semelhantes, a fim de verificar se as diferenças na composição do óleo devem-se a fatores ambientais ou a variação genética infraespecífica e se a floração influencia o rendimento e a composição do óleo. Os quimiotipos produtores de citral, carvona e linalol, foram denominados Lippia alba 1, 2 e 3, respectivamente. Os óleos essenciais foram extraídos por hidrodestilação de folhas e analisados por cromatografia com fase gasosa e cromatografia com fase gasosa acoplada ao espectrômetro de massas. O melhor rendimento foi obtido das plantas no estágio vegetativo. A composição do óleo essencial manteve-se inalterada para os três quimiotipos após cultivo em condições semelhantes e também não variou qualitativamente durante o crescimento vegetativo e floração. Os dados obtidos reforçam a idéia que as diferenças na composição do óleo essencial dos quimiotipos refletem variações genotípicas entre as plantas e que a extração de óleo essencial de $L$. alba deve ser efetuada na fase de crescimento vegetativo, quando é maior o rendimento do óleo e a porcentagem dos componentes majoritários.
\end{abstract}

Unitermos: Lippia alba, Verbenaceae, óleo essencial, citral, carvona, linalol.

ABSTRACT: "Analysis of the essential oil from leaves of three Lippia alba (Mill.) N. E. Br. (Verbenaceae) chemotypes cultivated on the same conditions". The composition of Lippia alba essential oil varies in a manner that different chemotypes are recognized. This work deals with the analysis of the essential oil from three chemotypes of L. alba from different regions of Brazil, cultivated in similar conditions, to verify if the differences in their chemical composition can be due to environmental conditions or may be due to infra-specific genetic variation. The influence of flowering was investigated regarding essential oil yield and composition. The chemotypes producing citral, carvone and linalol were denominated chemotypes 1,2 and 3, respectively. The essential oils were obtained by hydrodistillation of the leaves, at flowering and vegetative growth stages. Essential oils were analyzed by GC and GC/MS. A better yield was obtained from plants at vegetative stage. The composition of the essential oils remained unchanged after cultivation in the same conditions, and did not vary qualitatively during flowering and vegetative periods. Relative percentages of the major compounds changed during flowering period. Data obtained allow us to reinforce the idea that differences in the essential oil composition of the three chemotypes are due to genotypic variations and that the essential oil extraction during the vegetative period enables a better yield and higher percentages of the major compounds.

Keywords: Lippia alba, Verbenaceae, essential oil, citral, carvone, linalol.

\section{INTRODUÇÃO}

Lippia alba ocorre nas américas Central e do Sul, habitando praticamente todas as regiões do Brasil, onde é muito empregada como medicinal, pelas suas propriedades sedativa, carminativa, analgésica, espasmolítica e emenagoga (Zétola et al., 2002; Vale et al., 1999; Matos et al., 1996). A composição de seu óleo essencial apresenta variação quantitativa e qualitativa, levando à separação em quimiotipos (Matos 
et al.,1996; Frigheto et al., 1998; Zoghbi et al.,1998), os quais poderiam apresentar atividades farmacológicas distintas, bem como diferenças morfológicas (Matos, 1996; Corrêa, 1992). As variações na composição do óleo essencial e características morfológicas têm sido observadas dependendo da origem geográfica do material, o que levou à hipótese de que seriam conseqüência da influência de fatores ambientais. (Retamar, 1994; Zoghbi et al.,1998).

Este trabalho tem como objetivo a análise do óleo essencial de folhas de três quimiotipos de Lippia alba, provenientes de diferentes regiões do Brasil, cultivados em condições semelhantes, a fim de verificar se as diferenças na composição do óleo essencial devemse a fatores ambientais ou a variação genética infraespecífica. Adicionalmente, verificou-se se a floração influencia o rendimento e a composição do óleo.

\section{MATERIAL E MÉTODOS}

\section{Material vegetal}

Estacas enraizadas dos três quimiotipos de Lippia alba, denominados Lippia alba 1,2 e 3, os quais produzem como componente majoritário no óleo essencial o citral, a carvona e o linalol, respectivamente, foram plantadas no Horto do Departamento de Botânica - UFRJ, em um mesmo canteiro, em local parcialmente sombreado, com distância mínima de $30 \mathrm{~cm}$ entre elas, em covas de aproximadamente $20 \mathrm{~cm}$ de diâmetro, preenchidas com terra adubada com estrume de vaca, onde cresceram sob rega diária. Os quimiotipos são provenientes do estado do Rio de Janeiro (1), do estado do Ceará (2), e do estado de São Paulo (3), respectivamente e foram cedidos pelo Prof. Dr. Benjamin Gilbert, de Farmanguinhos/Fiocruz, Rio de Janeiro. Foram analisados três indivíduos de cada quimiotipo, com mais de 1 ano após o plantio.

Ramos férteis foram depositados, como exsicatas, no Herbário do Departamento de Botânica, Instituto de Biologia, UFRJ (RFA), registradas sob os números 29421, 29422 e 29423, respectivamente. A identificação foi feita pela Dra ${ }^{\text {a }}$. Fátima Regina Gonçalves Salimena, da Universidade Federal de Juiz de Fora (UFJF).

\section{Extração e análise dos óleos essenciais}

Os óleos essenciais foram extraídos por hidrodestilação, empregando extrator descrito na Farmacopéia Brasileira, IV edição, durante $2 \mathrm{~h}$, de mistura de folhas de três indivíduos de cada quimiotipo de L. alba cultivados no horto do Departamento de Botânica, perfazendo $100 \mathrm{~g}$ para cada quimiotipo. O óleo foi extraído em agosto de 2000, época em que as plantas encontravam-se em floração, e em março de 2001, quando as plantas encontravam-se em fase de crescimento vegetativo. O rendimento em óleo foi medido em $\mathrm{g} / \mathrm{g}$ de peso fresco. Os valores foram determinados à partir de amostra composta de 3 indivíduos de cada quimiotipo.

A análise por cromatografia com fase gasosa (CG) foi efetuada em aparelho em equipamento Agilent HP 5890, serie II, sob as seguintes condições experimentais: coluna capilar de sílica fundida com $5 \%$ de fenilmetilsilicona (PE-5) com $20 \mathrm{~m} \times 0.18 . \mathrm{mm}$ D. I., com $0.4 \mu \mathrm{m}$ de espessura de fase, fluxo de $1 \mathrm{~mL}$ $\min ^{-1}$ de hidrogênio como gás de arraste; temperatura do injetor de $250^{\circ} \mathrm{C}$; temperatura do detector (FID) $300^{\circ} \mathrm{C}$; aquecimento da coluna programado para $60^{\circ}-240^{\circ} \mathrm{C}$ at $3^{\circ} \mathrm{C} \mathrm{min}^{-1}$. Para cada quimiotipo e cada estádio de desenvolvimento foram analisadas amostras compostas do óleo de três indivíduos. Análise por CG/EM empregou detector de massas Agilent 5973 MSD acoplado a cromatógrafo a gás modelo 6890 , tendo Hélio como gás de arraste, mesma coluna e condições descritas acima A temperatura da interface foi de $240^{\circ} \mathrm{C}$, fonte de íons, $230^{\circ} \mathrm{C}(70 \mathrm{eV})$ e a faixa de massas de 40 a 400 uma. $\mathrm{O}$ tempo de retenção foi medido em minutos. Para cada quimiotipo foram analisadas amostras compostas do óleo de três indivíduos.

\section{RESULTADOS E DISCUSSÃO}

\section{Rendimento durante o crescimento vegetativo e floração}

Aquantidade de óleo essencial de folhas, extraído por hidrodestilação e medido em $\mathrm{g} / \mathrm{g}$ de peso fresco, variou de acordo com a época do ano (Tabela 1). O menor rendimento foi observado na época em que as plantas encontravam-se em floração. Resultados discrepantes foram observados por Gupta et al. (2002) em estudo com Artemisia annua, onde a maior porcentagem de óleo essencial foi obtida à partir de plantas em floração. Por outro lado, Atti et al. (2002) referem que em plantas de

Tabela 1. Rendimento médio em óleo essencial de folhas de Lippia alba (Mill) N. E. Br. coletadas durante o crescimento vegetativo e floração.

\begin{tabular}{|lll}
\hline & \multicolumn{2}{l}{ Rendimento médio(g/g de peso fresco) } \\
\cline { 2 - 3 } L. alba 1 & Floração & Crescimento vegetativo \\
L. alba 2 & $0,15 \%$ & $0,30 \%$ \\
L. alba 3 & $0,40 \%$ & $0,50 \%$ \\
\hline
\end{tabular}


Tabela 2. Componentes do óleo essencial de folhas de Lippia alba (Mill) N. E. Br., quimiotipos 1, 2 e 3, coletadas em agosto de 2000 quando as plantas encontravam-se em floração. TR= Tempo de retenção, \%= porcentagem relativa, IK C= Índice de Kovats calculado.

\begin{tabular}{|c|c|c|c|c|c|c|c|c|c|}
\hline \multirow[b]{2}{*}{ Componente } & \multicolumn{3}{|c|}{ L. alba 1} & \multicolumn{3}{|c|}{ L. alba 2} & \multicolumn{3}{|c|}{ L. alba 3} \\
\hline & TR & $\%$ & IK C & TR & $\%$ & IK C & TR & $\%$ & IK C \\
\hline 3-Z-Hexenol & 3,23 & 1,48 & 857 & - & - & - & 3,22 & 0,14 & 856 \\
\hline$\alpha$-Tujeno & 4,84 & 0,15 & 931 & 4,83 & 0,18 & 931 & 4,83 & 0,05 & 931 \\
\hline$\alpha$-Pineno & 5,03 & 0,32 & 939 & 5,03 & 0,12 & 938 & 5,03 & 0,07 & 938 \\
\hline Sabineno & 6,15 & 0,48 & 977 & 6,15 & 2,02 & 977 & 6,14 & 1,23 & 977 \\
\hline 1-Octen-3-ol & 6,36 & 3,26 & 983 & - & - & - & 6,25 & 0,05 & 980 \\
\hline 6-Metil-5-hepten-2-ona & 6,59 & 3,46 & 990 & - & - & - & 6,34 & 1,18 & 983 \\
\hline Mirceno & 6,66 & 2,34 & 992 & 6,65 & 0,40 & 992 & 6,64 & 0,38 & 992 \\
\hline$\alpha$-Terpineno & - & - & - & 7,54 & 0,10 & 1020 & - & - & - \\
\hline$p$-Cimeno & 7,81 & 0,33 & 1028 & - & - & - & - & - & - \\
\hline Limoneno & 7,97 & 0,25 & 1032 & 8,00 & 16,98 & 1033 & 7,96 & 0,27 & 1032 \\
\hline 1,8-Cineol & - & - & - & - & - & - & 8,16 & 5,96 & 1038 \\
\hline$Z$ - $\beta$-Ocimeno & 8,27 & 0,18 & 1041 & - & - & - & 8,26 & 0,06 & 1040 \\
\hline$E$ - $\beta$-Ocimeno & 8,66 & 0,51 & 1051 & 8,65 & 0,56 & 1051 & 8,65 & 0,56 & 1051 \\
\hline$\gamma$-Terpineno & - & - & - & 9,07 & 0,23 & 1062 & - & - & - \\
\hline cis-Hidrato de sabineno & - & - & - & - & - & - & 9,50 & 0,19 & 1073 \\
\hline trans- Hidrato de sabineno & - & - & - & 9,50 & 0,82 & 1073 & - & - & - \\
\hline trans-Óxido de linalol & - & - & - & - & - & - & 10,37 & 0,10 & 1093 \\
\hline Linalol & 10,52 & 0,07 & 1096 & 10,76 & 1,48 & 1102 & 10,98 & 60,05 & 1108 \\
\hline 1,3,8-para-Mentatrieno & - & - & - & - & - & - & 11,04 & 0,11 & 1110 \\
\hline cis-Verbenol & 12,36 & 0,14 & 1143 & 12,16 & 0,11 & 1138 & - & - & - \\
\hline Isobutirato de $Z$-3-hexenila & - & - & - & - & - & - & 12,53 & 0,19 & 1147 \\
\hline Citronelal & 12,72 & 0,14 & 1152 & 12,73 & 0,13 & 1152 & 12,73 & 0,12 & 1152 \\
\hline Óxido de $\beta$-pineno & 12,95 & 0,35 & 1157 & - & - & - & - & - & - \\
\hline Umbelulona & - & - & - & - & - & - & 13,62 & 0,33 & 1172 \\
\hline$\alpha$-Terpineol & - & - & - & - & - & - & 14,66 & 0,48 & 1194 \\
\hline cis-Diidro carvona & - & - & - & - & - & - & 14,87 & 0,04 & 1198 \\
\hline trans-Diidro carvona & - & - & - & - & - & - & 15,12 & 1,94 & 1204 \\
\hline trans-Carveol & - & - & - & - & - & - & 15,47 & 3,10 & 1213 \\
\hline Nerol & 16,41 & 5,48 & 1235 & - & - & - & - & - & - \\
\hline Citronelol & - & - & - & - & - & - & 16,37 & 0,21 & 1234 \\
\hline Neral & 17,02 & 25,82 & 1249 & - & - & - & 16,87 & 0,43 & 1246 \\
\hline Carvona & - & - & - & 17,19 & 52,8 & 1253 & - & - & - \\
\hline Geraniol & 17,63 & 9,2 & 1263 & - & - & - & 17,01 & 0,13 & 1249 \\
\hline Geranial & 18,38 & 33,98 & 1279 & - & - & - & 18,19 & 0,56 & 1275 \\
\hline$\alpha$-Copaeno & 22,87 & 0,27 & 1380 & - & - & - & 22,78 & 0,23 & 1378 \\
\hline Acetato de geranila & 23,21 & 0,27 & 1387 & - & - & - & - & - & - \\
\hline$\beta$-Bourboneno & - & - & - & 23,17 & 0,26 & 1386 & - & - & - \\
\hline$\beta$-Cubebeno & - & - & - & 23,41 & 0,22 & 1391 & 23,41 & 0,22 & 1391 \\
\hline$\beta$-Elemeno & 23,43 & 0,25 & 1392 & 23,50 & 0,41 & 1393 & 23,50 & 1,33 & 1393 \\
\hline E-Cariofileno & 24,70 & 1,74 & 1422 & 24,67 & 0,29 & 1421 & 24,70 & 3,15 & 1422 \\
\hline$\beta$-Gurjuneno & 25,09 & 0,10 & 1431 & 25,09 & 0,13 & 1431 & 25,08 & 0,14 & 1431 \\
\hline$\alpha$-Humuleno & - & - & - & - & - & - & 26,14 & 0,41 & 1456 \\
\hline cis-Muurola-4(14) 5 dieno & 26,15 & 0,12 & 1457 & - & - & - & - & - & - \\
\hline alo-Aromadendreno & 26,27 & 0,18 & 1459 & 26,46 & 0,29 & 1464 & 26,45 & 0,22 & 1464 \\
\hline Germacreno D & - & - & - & 27,33 & 5,94 & 1484 & 27,34 & 5,20 & 1484 \\
\hline Biciclogermacreno & - & - & - & - & - & - & 27,97 & 0,23 & 1498 \\
\hline$\alpha$-Muuruleno & - & - & - & 27,98 & 0,29 & 1498 & - & - & - \\
\hline Germacreno A & - & - & - & 28,14 & 0,15 & 1502 & 28,14 & 0,15 & 1502 \\
\hline$\beta$-Bisaboleno & - & - & - & 28,33 & 0,16 & 1507 & 28,34 & 0,55 & 1507 \\
\hline$\gamma$-Cadineno & 28,71 & 0,11 & 1516 & - & - & - & - & - & - \\
\hline$\delta$-Cadineno & 29,09 & 0,9 & 1526 & 28,85 & 0,17 & 1520 & 28,84 & 0,44 & 1520 \\
\hline$\alpha$-Cadineno & - & - & - & - & - & - & 29,74 & 0,14 & 1542 \\
\hline Elemol & - & - & - & 30,24 & 4,95 & 1555 & - & - & - \\
\hline Germacreno B & - & - & - & - & - & - & 30,49 & 2,61 & 1561 \\
\hline$E$-Nerolidol & 30,76 & 0,38 & 1567 & - & - & - & 30,76 & 0,38 & 1567 \\
\hline Germacreno D-4-ol & - & - & - & - & - & - & 31,26 & 0,14 & 1579 \\
\hline Total & - & 92,2 & - & - & 89,1 & - & - & 93,5 & - \\
\hline
\end{tabular}


Tabela 3. Componentes majoritários dos óleos essenciais de Lippia alba (Mill) N. E. Br., quimiotipo 1, extraídos por hidrodestilação de folhas coletadas em agosto de 2000, quando as plantas encontravam-se em floração, e em março de 2001, quando encontravam-se em fase de crescimento vegetativo.

\begin{tabular}{lcc}
\hline \multicolumn{1}{c}{ Componente } & \multicolumn{2}{c}{ Teor (\%) } \\
\cline { 2 - 3 } & Crescimento vegetativo & Floração \\
\hline Z-3-Hexenol & 0,94 & 1,48 \\
1-Octen-3-ol & 0,82 & 3,26 \\
6-Metil-5-hepten-2-ona & 0,72 & 3,46 \\
Mirceno & 0,45 & 2,34 \\
Nerol & 4,60 & 5,48 \\
Neral & 29,30 & 25,82 \\
Geraniol & 8,19 & 9,20 \\
Geranial & 42,77 & 33,98 \\
E-Cariofileno & 2,05 & 1,74 \\
\hline
\end{tabular}

Os valores foram obtidos através de análise de amostra composta do óleo obtido de folhas de três indivíduos

Tabela 4. Componentes majoritários dos óleos essenciais de Lippia alba (Mill) N. E. Br., quimiotipo 2, extraídos por hidrodestilação de folhas coletadas em agosto de 2000, quando as plantas encontravam -se em floração, e em março de 2001, quando encontravam-se em fase de crescimento vegetativo.

\begin{tabular}{lcc}
\hline \multicolumn{1}{c}{ Componente } & & Teor (\%) \\
\cline { 2 - 3 } & Crescimento vegetativo & Floração \\
\hline Sabineno & 1,32 & 2,02 \\
Limoneno & 15,00 & 16,98 \\
Carvona & 61,78 & 52,80 \\
Germacreno D & 4,25 & 5,94 \\
Elemol & 3,20 & 4,95 \\
\hline
\end{tabular}

Os valores foram obtidos através de análise de amostra composta do óleo obtido de folhas de três indivíduos

Tabela 5. Componentes majoritários dos óleos essenciais de Lippia alba (Mill) N. E. Br., quimiotipo 3, extraídos por hidrodestilação de folhas coletadas em agosto de 2000, quando as plantas encontravam-se em floração, e em março de 2001, quando encontravam-se em fase de crescimento vegetativo.

\begin{tabular}{lcc}
\hline \multicolumn{1}{c}{ Componente } & \multicolumn{2}{c}{ Teor (\%) } \\
\cline { 2 - 3 } & Crescimento vegetativo & Floração \\
\hline Sabineno & 0,77 & 1,23 \\
6-Metil-5-hepten-2-ona & 0,54 & 1,18 \\
1,8-Cineol & 4,22 & 5,96 \\
Linalol & 73,99 & 60,05 \\
trans-Diidro carvona & 1,86 & 1,94 \\
trans-Carveol & 1,99 & 3,10 \\
B-Elemeno & 2,82 & 1,33 \\
E-Cariofileno & 2,78 & 3,15 \\
Germacreno D & 1,73 & 5,20 \\
Germacreno B & 0,72 & 2,61 \\
\hline
\end{tabular}

Os valores foram obtidos através de análise de amostra composta do óleo obtido de folhas de três indivíduos

L. alba coletadas em Caxias do Sul o maior rendimento em óleo ocorreu no período de dezembro a março. Como este é o período em que a espécie encontra-se em fase de crescimento vegetativo e, sabendo que a relação entre fase de crescimento e produção de óleo essencial é variável segundo a espécie estudada, podemos concluir que em L. alba a maior produção de óleo ocorre fora do período de floração.
Os valores foram obtidos utilizando-se, para cada quimiotipo, em cada estádio de desenvolvimento, amostras compostas do óleo obtido de folhas de três indivíduos.

Composição do óleo essencial analisado por cromatografia com fase gasosa 
Os resultados das análises dos óleos essenciais de $L$. alba 1, 2 e 3 estão descritos na Tabela 2. Para L. alba 1 foram identificados 29 componentes, os quais representam $92,2 \%$ do óleo. Em L. alba 2 os 26 componentes identificados perfazem $89,1 \%$ do óleo. Em L. alba 3 foram identificados 42 constituintes, representando $93,5 \%$ do óleo.

A observação da Tabela 2 nos permite concluir que a diversidade na composição do $\mathrm{OE}$ dos quimiotipos estudados não se deve a fatores ambientais, já que plantas cultivadas lado a lado, em um mesmo canteiro, por mais de um ano, mantiveram a composição original. A diferença deve-se provavelmente à diversidade no genótipo das plantas. De fato, resultados preliminares de estudo citogenético demonstraram que o número cromossômico de pelo menos dois dos quimiotipos é diferente, o que denota processo de especiação (Tavares et al, 2003).

Os valores foram obtidos utilizando-se, para cada quimiotipo, análise de amostras compostas do óleo obtido de folhas de três indivíduos.

\section{Análise do óleo essencial durante o crescimento vegetativo e floração}

A análise dos óleos essenciais obtidos na época em que as plantas encontravam-se em floração e dos óleos extraídos anteriormente, quando a floração ainda não havia tido início, nos permite concluir que não houve variação qualitativa dos componentes majoritários nos dois períodos de vida das plantas (Tabelas 3, 4 e 5). Por outro lado a análise quantitativa destes elementos nos mostra que a porcentagem de citral (geranial + neral) carvona e linalol sofreu uma ligeira diminuição durante a época de floração (Tabelas 3,4 e 5) ao passo que houve aumento na porcentagem do limoneno. Variações quantitativas na composição do óleo essencial durante a fase de crescimento vegetativo e a floração também foram observadas em Artemisia campestris var. glutinosa (Juteau et al., 2002) e Artemísia annua (Gupta et al., 2002).

\section{CONCLUSÕES}

Os dados obtidos no presente estudo reforçam a idéia de que as diferenças na composição do óleo essencial dos três quimiotipos não constituem produto da influência de fatores ambientais mas refletem variação genotípica entre as plantas já que tais diferenças mantiveram-se em plantas cultivadas lado a lado em um mesmo canteiro. Permitem-nos ainda concluir que a extração de óleo essencial de $L$. alba para a obtenção de citral carvona e linalol deve ser efetuada em plantas em fase de crescimento vegetativo quando o rendimento do óleo e os teores dos componentes majoritários são maiores.

\section{REFERÊNCIAS}

Atti SL, Pansera MR, Atti SAC, Rossato M, Pauletti GF, Rotal D, Paroul N, Moyna P 2002. Variation in essential oil yield and composition of Lippia alba (Mill.) N. E. Br. grown in southern Brazil. Rev Bras Pl Med 4: 72-74.

Corrêa CBV, 1992. Contribuição ao estudo de Lippia alba (Mill.) N. E. Br. ex Britt \& Wilson - erva-cidreira. Rev Bras Farm 73: 57-64.

Frighetto N, Oliveira JG, Siani AC, Chagas KC 1998. Lippia alba Mill N. E. Br. (Verbenaceae) as a source of linalool. J Essent Oil Res 10: 578-580.

Gupta SK, Singh P, Bajpai P, Ram G, Digvijai S, Gupta MM, Jain DC, Khanuja SPS, Kumar S 2002. Morphogenetic variation for artemisin and volatile oil in Artemisia annua. Ind Crop Prod 16: 2217-2224.

Juteau F, Masotti V, Bessiére JM, Viano J 2002. Compositional characteristics of the essential oil of Artemisia campestris var. glutinosa. Biochem Syst Ecol 30: 1065-1070.

Matos FJA 1996. As ervas cidreiras do Nordeste do Brasil. Estudo de três quimiotipos de Lippia alba (Mill.) N. E. Brown (Verbenaceae) Parte II - Farmacoquímica. Rev Bras Farm 77: 137-141.

Matos FJA, Machado MIL, Craveiro AA, Alencar JW 1996. Essential oil composition of two chemotypes of Lippia alba grown in northeast Brazil. J Essent Oil Res 8: 695-698.

Retamar JA 1994. Variaciones fitoquimicas de la especie Lippia alba (salvia morada) y sus aplicaciones en la quimica fina. Essenze Derivati Agrumari 16: 55-60.

Tavares ES, Julião LS, Leitão SG, Lage CLS, Viccini L, Pierre PMO, Davide LC 2003. Diferenças morfológicas, anatômicas e fisiológicas de dois quimiotipos de Lippia alba (Mill) N. E. Br. relacionadas à poliploidia. VI Congresso de Ecologia do Brasil, Simpósios Biodiversidade, Unidades de Conservação, Indicadores Ambientais, Cerrado e Caatinga. Fortaleza: Editora da Universidade Federal do Ceará, 99.

Vale TG, Matos FJA, Lima TCM, Viana, GSB 1999. Behavioral effects of essential oils from Lippia alba (Mill) N. E. Brown chemotypes. J Ethnopharmacol 67: 127-133.

Zétola M, Lima TCM, Sonaglio D, Gonzáles-Ortega G, Limberger RP, Petrovick PR, Bassani VL 2002. CNS activities of liquid and spray-dried extracts from Lippia alba - Verbenaceae (Brasilian false melissa). J Ethnopharmacol 82: 207-215.

Zoghbi MGB, Andrade EHA, Santos AS, Silva MH, Maia JGS 1998. Essential oils of Lippia alba (Mill) N. E. Br growing wild in the Brazilian Amazon. Flavour Frag J 13: 47-48. 